\title{
Secrecy Rate and Harvested Energy Trade-off for MISO Channels with Finite-Alphabet Inputs
}

\author{
Sina Rezaei Aghdam and Tolga M. Duman \\ Dept. of Electrical and Electronics Engineering \\ Bilkent University, Ankara, Turkey, TR 06800 \\ Emails: \{aghdam,duman\}@ee.bilkent.edu.tr
}

\begin{abstract}
We focus on transmit signal design for multipleinput single-output (MISO) wiretap channels with simultaneous wireless information and power transfer (SWIPT). Assuming that the channel inputs are drawn from standard constellation sets, we formulate secrecy rate maximization problems subject to power and harvested energy constraints. We tackle these problems under two different assumptions on the channel state information (CSI) at the transmitter. First, we consider a scenario in which the transmitter knows the CSI for both the information receiver and the energy receiver (potential eavesdropper), and we propose a precoder optimization approach. Then, we investigate the case where only perfect CSI of the information receiver is available along with the statistical CSI of the energy receiver. Our numerical results demonstrate the efficacy of the proposed solutions.
\end{abstract}

\section{INTRODUCTION}

Simultaneous wireless information and power transfer (SWIPT) is a promising solution for mitigating the energy scarcity in energy constrained networks. Despite various opportunities SWIPT provides for self-sustainability of the wireless communication systems, ensuring confidentiality of the transmitted data remains as a major concern. This is particularly due to the fact that deployment of a SWIPT system is typically in a manner that the energy receiver (ER) is closer to the source than the information receiver (IR), and hence, the ER usually enjoys a better channel quality.

To overcome the above concern, many different solutions are proposed (see [1] for a survey). Among these solutions, multiple-antenna techniques are shown to exhibit a high potential for enhancing security in SWIPT systems. The secrecy rate of the SWIPT multiple input multiple output (MIMO) wiretap channel is studied in [2] and [3]. In [2], the authors assume that the transmitter knows only the statistics of the channels, and after deriving an asymptotic expression for the ergodic secrecy rate, they obtain the optimal input structure, which achieves the trade-off between ergodic secrecy rate and harvested energy. On the other hand, the secrecy rates of MIMO SWIPT wiretap channels are characterized under a global channel state information (CSI) assumption in [3].

The aforementioned characterizations and designs rely on a Gaussian input assumption. However, practical communication systems employ discrete signaling and the channel inputs are drawn from standard constellations such as phase shift keying (PSK) and quadrature amplitude modulation (QAM). While the impact of finite-alphabet inputs on the trade-off between the mutual information of the IR and the harvested energy at the ER is explored in [4], to the best of our knowledge, there is no study on secure SWIPT under the finite-alphabet input assumption, motivating this work.

In this paper, we consider a multiple input single output (MISO) SWIPT wiretap channel where we assume that the CSI of the IR is perfectly known by the transmitter. Regarding the availability of the ER's CSI at the transmitter (ECSIT), we separately study the scenarios with perfect and statistical channel knowledge. For each scenario, we first review the optimal solutions for 1) maximizing the secrecy rates without taking into account the harvested energy at the ER, and 2) maximizing the harvested energy at the ER without considering the secrecy requirement. Then, we introduce a transmit signal design algorithm for achieving the trade-off between the secrecy rate and the harvested energy. The proposed algorithm relies on precoding and artificial noise injection. While, in the context of physical layer security with no energy harvesting constraints, the artificial noise is typically designed to be injected in the null-space of the main channel [5], such a strategy is not as effective when harvested energy is also of concern. Hence, we borrow the idea of injecting generalized artificial noise from [6], and introduce a framework for jointly optimizing the data and artificial noise precoders such that the ergodic secrecy rate is maximized under the constraint that the harvested energy exceeds a predefined threshold.

Performing a full search for the optimal data precoder and artificial noise covariance matrices possesses a high computational complexity. As a low complexity alternative, we also propose a two-stage precoder design algorithm where we first obtain the data precoder without considering the energyharvesting constraint. Under the assumption that the ER is operating at high SNRs, only a fraction of the total power is allocated to data transmission. Therefore, in the second stage, a simple power allocation strategy is proposed, which divides the remaining power between two signals: an artificial noise signal, which is transmitted along the null-space of the IR's channel, and an energy signal, which is beamformed towards the ER's direction.

The rest of this paper is organized as follows. Section II describes the system model and provides the problem formulation. The trade-off between the secrecy rate and the 
harvested energy and also our proposed transmit signal design algorithms under perfect and statistical ECSIT are presented in Sections III and IV, respectively. In Section V, we describe the proposed low complexity two-stage precoder design approach. The numerical examples are given in Section VI, and the paper is concluded in Section VII.

Notation: Vectors and matrices are denoted with the lowercase and uppercase bold letters, respectively. $\mathbb{E}_{a}\{$.$\} denotes$ the expectation over a random variable $a$ and the positive part of a real quantity $b$ is denoted by $(b)^{+}=\max \{b, 0\} .()^{H}$, $(.)^{*}$ and $\|.\|_{F}$ represent Hermitian, complex conjugate and Frobenius norm operations, respectively.

\section{System Model And PRoblem Formulation}

Consider a multi-antenna transmitter with $N_{t}$ antenna elements whose objective is to deliver data bits to an intended single-antenna IR and to transfer energy to a single-antenna ER. The received signals at the IR and the ER are given by

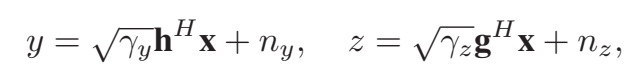

respectively, where the vectors $\mathbf{h}^{H} \in \mathbb{C}^{1 \times N_{t}}$ and $\mathbf{g}^{H} \in \mathbb{C}^{1 \times N_{t}}$ denote the channels corresponding to the IR and the ER, respectively. The distance-dependent path loss over these channels are represented by the coefficients $\gamma_{y}$ and $\gamma_{z}$. The $N_{t} \times 1$ vector $\mathbf{x}$ is the channel input, and $n_{y}$ and $n_{z}$ stand for circularly symmetric complex additive white Gaussian noise terms, which follow $\mathcal{C N}\left(0, \sigma_{n_{y}}^{2}\right)$ and $\mathcal{C N}\left(0, \sigma_{n_{z}}^{2}\right)$, respectively.

Regarding the availability of the ECSIT, we consider two scenarios. In the first case, perfect CSI on both channels is available at the transmitter, and we assume that the channels are independent Rayleigh fading, i.e., we model the channels using vectors, the elements of which are independent and identically distributed (i.i.d.) zero-mean and unit-variance circularly symmetric complex Gaussian random variables $\mathcal{C N}(0,1)$. In the second case, perfect IR CSI and statistical ER CSI are available. In this case, we assume that the IR's channel is an independent Rayleigh fading channel, however, a transmit correlation exists for the ER's channel, namely [2]

$$
\mathbf{g}^{H}=\hat{\mathbf{g}}^{H} \boldsymbol{\Psi}_{t}^{1 / 2}
$$

where $\hat{\mathbf{g}} \in \mathbb{C}^{1 \times N_{t}}$ is a complex vector with i.i.d. zero mean unit variance Gaussian entries, and $\boldsymbol{\Psi}_{t} \in \mathbb{C}^{N_{t} \times N_{t}}$ is the correlation matrix, which can be acquired by the transmitter.

For both scenarios, it is assumed that the ER can act as a potential eavesdropper for the confidential data transmitted to the IR. Our objective is to maximize the number of bits transmitted to the IR while simultaneously guaranteeing that the ER is ignorant about the transmitted information and the harvested power of the ER is larger than a predefined value. The transmit signal is constructed as

$$
\mathbf{x}=\mathbf{P}_{D} \mathbf{s}+\mathbf{u}
$$

where $\mathbf{s} \in \mathbb{C}^{N_{t} \times 1}$ is the information vector with zero mean and identity covariance matrix, the elements of which are assumed to be drawn from standard constellations such as PSK and QAM. $\mathbf{P}_{D} \in \mathbb{C}^{N_{t} \times N_{t}}$ denotes the data precoder matrix. $\mathbf{u}$ is a circularly symmetric complex Gaussian signal, which follows $\mathcal{C N}\left(0, \mathbf{K}_{\mathbf{u}}\right)$ playing the dual role of the artificial noise and the energy signal.

The IR's and the ER's channels are both ergodic. The channel gains are assumed to be fixed during each coherence interval while they change independently from one coherence interval to the next. Moreover, we assume that the coherence intervals are long enough so that random coding arguments can be invoked. Accordingly, an achievable ergodic secrecy rate is given by [7]

$$
\bar{R}_{s}=\mathbb{E}_{\mathbf{h}, \mathbf{g}}\left\{(I(\mathbf{s} ; y \mid \mathbf{h})-I(\mathbf{s} ; z \mid \mathbf{g}))^{+}\right\}
$$

where the mutual information for the IR's channel is given by

$$
\begin{gathered}
I(\mathbf{s} ; y \mid \mathbf{h})=N_{t} \log M-\frac{1}{M^{N_{t}}} \\
\times \sum_{i=1}^{M^{N_{t}}} \mathbb{E}_{n_{y}^{\prime}}\left\{\log \sum_{j=1}^{M^{N_{t}}} \exp \left(-\frac{\left|\sqrt{\gamma_{y}} \mathbf{h}^{H} \mathbf{P}_{D} \mathbf{d}_{i j}+n_{y}^{\prime}\right|^{2}-\left|n_{y}^{\prime}\right|^{2}}{\sigma_{n_{y}^{\prime}}^{2}+\gamma_{y} \mathbf{h}^{H} \mathbf{K}_{\mathbf{u}} \mathbf{h}}\right)\right\},
\end{gathered}
$$

with $\mathbf{d}_{i j}=\mathbf{s}_{i}-\mathbf{s}_{j}$ and $n_{y}^{\prime}=\sqrt{\gamma_{y}} \mathbf{h}^{H} \mathbf{u}+n_{y}$. The mutual information over the ER's channel, i.e., $I(\mathbf{s} ; z \mid \mathbf{g})$, can be calculated in exactly the same way.

The average harvested energy at the ER is given by

$$
\bar{E}_{H}=\epsilon \gamma_{z} \mathbb{E}_{\mathbf{g}}\left\{\mathbf{g}^{H} \mathbf{K}_{\mathbf{x}} \mathbf{g}\right\}
$$

where $\mathbf{K}_{\mathbf{x}}$ is the covariance matrix of the channel input $\mathbf{x}$. The constant $\epsilon$ in (6) denotes the energy conversion rate. For convenience, without loss of generality, we assume that $\epsilon=1$ as in [2].

Throughout the paper, we provide approaches for joint optimization of the precoder matrix $\mathbf{P}_{D}$ and the covariance matrix $\mathbf{K}_{\mathbf{u}} \in \mathbb{C}^{N_{t} \times N_{t}}$ such that the ergodic secrecy rate is maximized subject to a power constraint while guaranteeing that the harvested energy at the ER is larger than a given value. To do this, we utilize the lower bounds on the instantaneous secrecy rates under the perfect and statistical ECSIT given by

$$
\begin{gathered}
R_{s, l}=I(\mathbf{s} ; y \mid \mathbf{h})-I(\mathbf{s} ; z \mid \mathbf{g}), \\
R_{s, l}^{\prime}=I(\mathbf{s} ; y \mid \mathbf{h})-\mathbb{E}_{\mathbf{g}} I(\mathbf{s} ; z \mid \mathbf{g}),
\end{gathered}
$$

respectively. Under the full CSI assumption, we use the harvested power conditioned on $\mathbf{g}$, i.e.,

$$
E_{H}=\gamma_{z}\left(\mathbf{g}^{H} \mathbf{P}_{D} \mathbf{P}_{D}^{H} \mathbf{g}+\mathbf{g}^{H} \mathbf{K}_{\mathbf{u}} \mathbf{g}\right),
$$

as our design metric. It should be noted that in (9), $\mathbf{g}$ stands for the realization of the ER's channel. Then, the relevant optimization problem when perfect ECSIT is available becomes

$$
\begin{array}{ll} 
& \max _{\mathbf{P}_{D}, \mathbf{K}_{\mathbf{u}}} R_{s, l} \\
\text { s.t. } \quad \operatorname{tr}\left(\mathbf{P}_{D} \mathbf{P}_{D}^{H}\right)+\operatorname{tr}\left(\mathbf{K}_{\mathbf{u}}\right) \leq N_{t} P_{t x} \text { and } E_{H} \geq E_{0} .
\end{array}
$$

Similarly, under the statistical ECSIT, the relevant optimization problem is expressed as

$$
\begin{gathered}
\max _{\mathbf{P}_{D}, \mathbf{K}_{\mathbf{u}}} R_{s, l}^{\prime} \\
\text { s.t. } \operatorname{tr}\left(\mathbf{P}_{D} \mathbf{P}_{D}^{H}\right)+\operatorname{tr}\left(\mathbf{K}_{\mathbf{u}}\right) \leq N_{t} P_{t x} \text { and } \bar{E}_{H} \geq E_{0},
\end{gathered}
$$


where the constraint is on the average harvested power $\bar{E}_{H}$ defined in (6).

\section{Secrecy Rate-Harvested Energy TRade-OfF UNDER PERFECT CSIT}

In this section, we characterize the trade-off between the ergodic secrecy rate and the harvested energy for the scenarios with perfect CSIT. The assumption of availability of the perfect CSI of the ER at the transmitter is reasonable when the ER is a legitimate user of the network. After reviewing the solutions to the problems of maximizing the secrecy rates without considering the harvested energy constraint and also maximizing the harvested energy without taking into account the secrecy requirement, we introduce an iterative approach for solving the optimization problem in (10)-(11).

We define the signal $\mathbf{u}$ in (3) as

$$
\mathbf{u}=\mathbf{P}_{E} \mathbf{u}_{E},
$$

where $\mathbf{P}_{E} \in \mathbb{C}^{N_{t} \times N_{t}}$ and $\mathbf{u}_{E} \sim \mathcal{C N}\left(0, \mathbf{I}_{N_{t}}\right)$, and therefore, $\mathbf{K}_{\mathbf{u}}=\mathbf{P}_{E} \mathbf{P}_{E}^{H}$, i.e., the optimization of the covariance matrix $\mathbf{K}_{\mathbf{u}}$ reduces to the optimization of $\mathbf{P}_{E}$. In the following, we discuss the design of the $\mathbf{P}_{D}$ and $\mathbf{P}_{E}$ matrices for achieving the secrecy rate-harvested energy trade-off.

Proposition 1. [From [9]] Under the assumption that both receivers operate in the high-SNR regime, with perfect CSIT, the maximum secrecy rate is achieved by data precoders in the following form

$$
\boldsymbol{P}_{D}=\boldsymbol{W}_{g} \tilde{\boldsymbol{P}}_{D}
$$

where $\boldsymbol{W}_{g} \in \mathbb{C}^{N_{t} \times N_{t}-1}$ is an orthonormal basis for the nullspace of $\boldsymbol{g}^{H}$ and $\tilde{\boldsymbol{P}}_{D} \in \mathbb{C}^{N_{t}-1 \times N_{t}}$ is the precoder matrix, which maximizes the mutual information over the effective channel $\boldsymbol{h}^{H} \boldsymbol{W}_{g}$ under the power constraint $\operatorname{tr}\left(\tilde{\boldsymbol{P}}_{D} \tilde{\boldsymbol{P}}_{D}^{H}\right) \leq$ $N_{t} P_{t x}$

Proposition 2. [From [10]] When the instantaneous CSI of the ER is available at the transmitter, the optimal transmission strategy for maximizing the harvested power is to use all the available power for transmitting $\boldsymbol{u}$ in (14) with the precoders of the form $\boldsymbol{P}_{E}=\left[\begin{array}{ll}\tilde{\boldsymbol{P}}_{E} & \boldsymbol{0}_{N_{t} \times N_{t}-1}\end{array}\right]_{N_{t} \times N_{t}}$ where

$$
\tilde{\boldsymbol{P}}_{E}=\sqrt{N_{t} P_{t x}} \frac{\boldsymbol{g}}{\|\boldsymbol{g}\|} \text {. }
$$

Propositions 1 and 2 reveal that the maximization of the secrecy rate and maximization of the harvested energy are two conflicting objectives. This is because, by transmitting in the null-space of the eavesdropper's channel (which is secrecy capacity achieving according to Proposition 1), no power is harvested at the ER. Meanwhile, the secrecy rate drops to zero when (only) beamforming the artificial noise (energy signal) in the direction of the ER. Therefore, a solution that provides a good trade-off lies between these two approaches.

In the following, we introduce a framework for joint optimization of $\mathbf{P}_{D}$ and $\mathbf{P}_{E}$. In an attempt to solve the optimization

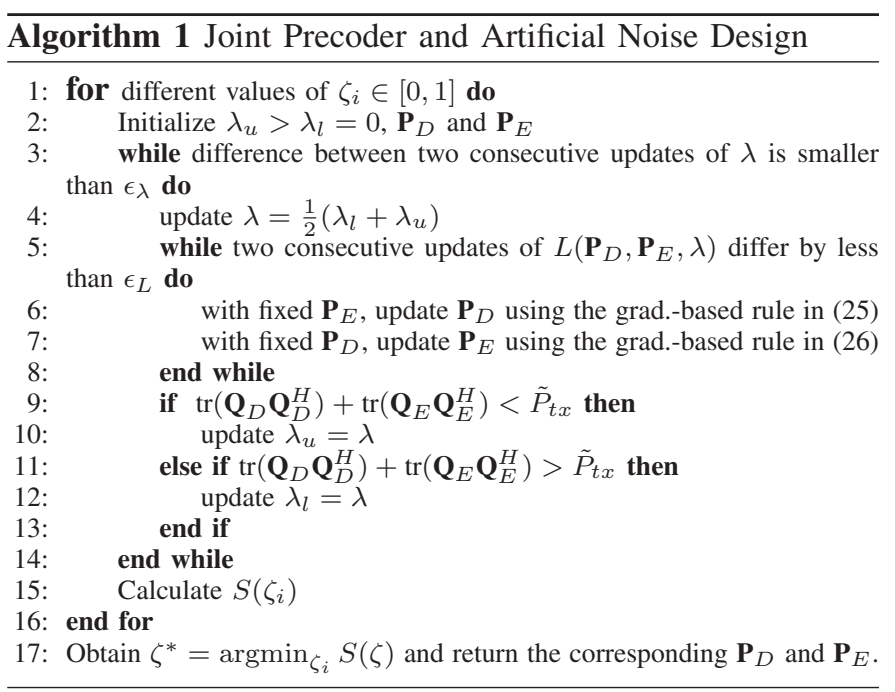

problem in (10)-(11), we formulate a surrogate dual problem in which we combine the two constraints in (11) as

$$
S(\zeta)=\max _{\mathbf{P}_{D}, \mathbf{P}_{E}} R_{s, l}
$$

s.t. $(1-\zeta)\left(\operatorname{tr}\left(\mathbf{P}_{D} \mathbf{P}_{D}^{H}\right)+\operatorname{tr}\left(\mathbf{P}_{E} \mathbf{P}_{E}^{H}\right)\right)-\zeta E_{H} \leq(1-\zeta) N_{t} P_{t x}-\zeta E_{0}$,

where $0 \leq \zeta \leq 1$ and $R_{s, l}$ and $E_{H}$ are as given in (7) and (9), respectively. The surrogate dual problem is defined as [11], [4]

$$
S\left(\zeta^{*}\right)=\min _{\zeta} S(\zeta) .
$$

By defining matrix $\mathbf{C}=\left((1-\zeta) \mathbf{I}_{N_{t}}-\zeta \mathbf{g g}^{H}\right)^{\frac{1}{2}}$ and $\tilde{P}_{t x}=(1-\zeta) N_{t} P_{t x}-\zeta E_{0}$, given that $\frac{\zeta}{1-\zeta}<\frac{1}{\beta}$, where $\beta$ is the maximal eigenvalue of $\mathbf{g g}^{H}$, the optimization problem in (17)-(18) can be simplified to

$$
\begin{gathered}
S(\zeta)=\max _{\mathbf{P}_{D}, \mathbf{P}_{E}} R_{s, l} \\
\text { s.t. } \quad \operatorname{tr}\left(\mathbf{Q}_{D} \mathbf{Q}_{D}^{H}\right)+\operatorname{tr}\left(\mathbf{Q}_{E} \mathbf{Q}_{E}^{H}\right) \leq \tilde{P}_{t x},
\end{gathered}
$$

where $\mathbf{Q}_{D}=\mathbf{C} \mathbf{P}_{D}$ and $\mathbf{Q}_{E}=\mathbf{C} \mathbf{P}_{E}$.

In order to solve (20)-(21), we define a Lagrange dual optimization problem as follows. The Lagrangian of (20) is given as:

$$
L\left(\mathbf{P}_{D}, \mathbf{P}_{E}, \lambda\right)=R_{s, l}+\lambda\left(\tilde{P}_{t x}-\operatorname{tr}\left(\mathbf{Q}_{D} \mathbf{Q}_{D}^{H}\right)+\operatorname{tr}\left(\mathbf{Q}_{E} \mathbf{Q}_{E}^{H}\right)\right),
$$

where $\lambda$ is the Lagrange dual variable associated with the constraint in (21). The associated dual optimization problem is defined as

$$
\min _{\lambda>0} T(\lambda)
$$

where

$$
T(\lambda)=\max _{\mathbf{P}_{D}, \mathbf{P}_{E}} L\left(\mathbf{P}_{D}, \mathbf{P}_{E}, \lambda\right) .
$$

In order to solve (23), we employ a bisection method as explained in Algorithm 1. For each value of $\lambda$, we optimize $\mathbf{P}_{D}$ and $\mathbf{P}_{E}$ in an alternating fashion. The optimization of $\mathbf{P}_{D}$ with a fixed $\mathbf{P}_{E}$ and also the optimization of $\mathbf{P}_{E}$ with a fixed 
$\mathbf{P}_{D}$ are carried out using gradient descent based approaches. Particularly, with a fixed $\mathbf{P}_{E}$, the optimal $\mathbf{P}_{D}$ is updated by taking steps proportional to the gradient of the objective function as

$\mathbf{P}_{D}(k+1)=\left[\mathbf{P}_{D}(k)+\mu \nabla_{\mathbf{P}_{D}} R_{s, l}(k)\right]_{\operatorname{tr}\left(\mathbf{Q}_{D} \mathbf{Q}_{D}^{H}\right) \leq \tilde{P}_{t x}-\operatorname{tr}\left(\mathbf{Q}_{E} \mathbf{Q}_{E}^{H}\right)}^{\dagger}$,

where $\mu$ denotes the step size of the update and

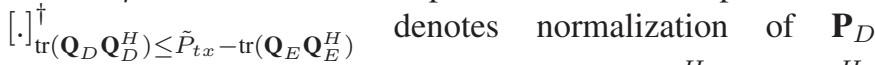
and $\mathbf{P}_{E}$ (while keeping the ratio $\operatorname{tr}\left(\mathbf{P}_{D} \mathbf{P}_{D}^{H}\right) / \operatorname{tr}\left(\mathbf{P}_{E} \mathbf{P}_{E}^{H}\right)$ at the same level prior to normalization) such that $\operatorname{tr}\left(\mathbf{Q}_{D} \mathbf{Q}_{D}^{H}\right) \leq \tilde{P}_{t x}-\operatorname{tr}\left(\mathbf{Q}_{E} \mathbf{Q}_{E}^{H}\right) \quad$ is satisfied. Similarly, for optimizing $\mathbf{P}_{E}$ with a fixed $\mathbf{P}_{D}$, we use

$\mathbf{P}_{E}(k+1)=\left[\mathbf{P}_{E}(k)+\mu \nabla_{\mathbf{P}_{E}} R_{s, l}(k)\right]_{\operatorname{tr}\left(\mathbf{Q}_{E} \mathbf{Q}_{E}^{H}\right) \leq \tilde{P}_{t x}-\operatorname{tr}\left(\mathbf{Q}_{D} \mathbf{Q}_{R}^{H}\right)}^{\dagger}$

The derivations of the gradients $\nabla_{\mathbf{P}_{D}} R_{s, l}$ and $\nabla_{\mathbf{P}_{E}} R_{s, l}$ are provided in Appendix A.

In order to obtain the solution for (19), we perform a one-dimensional search over multiple values of $\zeta$. One may note that the solution of the surrogate dual problem may not always be feasible in the original problem. When this is the case, the optimization procedure should be repeated with new constraints (e.g., smaller values of $E_{0}$ ) until the obtained solution satisfies the original constraints.

\section{Secrecy Rate-Harvested Energy Trade-off WITH STATISTICAL ECSIT}

We now focus on the scenarios where only statistical information on the ER's channel is available at the transmitter. More specifically, we consider a correlated channel towards the ER as in (2), and assume that the transmitter knows only the transmit correlation matrix $\Psi_{t}$.

Similar to Section III, we first review the existing solutions for maximizing the secrecy rate without any harvested energy constraint as well as the harvested energy maximization without any secrecy constraint. No closed-form optimal solution is available for the former problem. However, different numerical approaches are available in the literature, e.g., [12], [9] and [13], which facilitate obtaining precoder matrices $\mathbf{P}_{D}$ and $\mathbf{P}_{E}$ as suboptimal solutions. As for the latter problem, the optimal transmit signal for maximization of the harvested energy without secrecy constraints is as given in the following proposition.

Proposition 3. [From [2]] When the transmitter knows only the statistical CSI of the ER, the optimal transmission strategy for maximizing the harvested energy is to use all the available power for transmitting $\boldsymbol{u}$ on the strongest eigenmode of $\boldsymbol{\Psi}_{t}$. In other words, if we consider the eigenvalue decomposition of the ER's channel as $\boldsymbol{\Psi}_{t}=\boldsymbol{V}_{\boldsymbol{\Psi}} \boldsymbol{\Sigma}_{\boldsymbol{\Psi}} \boldsymbol{V}_{\boldsymbol{\Psi}}^{H}$, the optimal artificial noise (energy signal) precoder matrix is $\boldsymbol{P}_{E}=\left[\begin{array}{ll}\tilde{\boldsymbol{P}}_{E} & \boldsymbol{0}_{N_{t} \times N_{t}-1}\end{array}\right]_{N_{t} \times N_{t}}$ where

$$
\tilde{\boldsymbol{P}}_{E}=\sqrt{N_{t} P_{t x}} \boldsymbol{v}_{\boldsymbol{\Psi}_{1}}
$$

with $\boldsymbol{v}_{\boldsymbol{\Psi}_{1}}$ denoting the first column of $\boldsymbol{V}_{\boldsymbol{\Psi}}$.
Our objective is to solve the optimization problem in (12)(13). To do this, we employ a similar dual optimization as introduced in Section III by simply replacing $R_{s, l}$ and $E_{H}$ in (17)-(18) by the expressions given in (8) and (6), respectively. Namely, the optimization problem in (20)-(21) is rewritten as

$$
\begin{gathered}
S(\zeta)=\max _{\mathbf{P}_{D}, \mathbf{P}_{E}} R_{s, l}^{\prime} \\
\text { s.t. } \quad \operatorname{tr}\left(\mathbf{Q}_{D}^{\prime} \mathbf{Q}_{D}^{\prime H}\right)+\operatorname{tr}\left(\mathbf{Q}_{E}^{\prime} \mathbf{Q}_{E}^{\prime H}\right) \leq \tilde{P}_{t x},
\end{gathered}
$$

where $\mathbf{Q}_{D}^{\prime}=\left((1-\zeta) \mathbf{I}_{N_{t}}-\zeta \Psi_{t}\right)^{\frac{1}{2}} \mathbf{P}_{D}$ and $\mathbf{Q}_{E}^{\prime}=$ $\left((1-\zeta) \mathbf{I}_{N_{t}}-\zeta \mathbf{\Psi}_{t}\right)^{\frac{1}{2}} \mathbf{P}_{E}$. This problem is solved using the same steps given in Algorithm 1 by simply replacing $\mathbf{Q}_{D}$, $\mathbf{Q}_{E}$ and $R_{s, l}$ with $\mathbf{Q}_{D}^{\prime}, \mathbf{Q}_{E}^{\prime}$ and $R_{s, l}^{\prime}$, respectively.

\section{Low Complexity Two-Stage Precoder Design}

Due to the nature of SWIPT systems, the ER is expected to work at high SNRs. As a result, without taking into account the harvested energy constraint, existing transmit signal design algorithms over MISO wiretap channels with finite-alphabet inputs with both perfect and statistical CSI of the ER (see e.g., [9] for the perfect and [12], [13] for the statistical ECSIT scenario) lead to solutions, which allocate only a small fraction of total power for data transmission. The reason behind this observation is that, with a higher power transmission, the eavesdropper would be able to decode the transmitted data. In this section, we take advantage of this property to propose a low complexity alternative for the schemes given in Sections III and IV.

Let us first consider the full CSI case. In the proposed low complexity approach, rather than optimizing the covariance matrix $\mathbf{K}_{\mathbf{u}}$, we propose to construct $\mathbf{u}$ in (3) as

$$
\mathbf{u}=\alpha_{A N}^{2} \mathbf{W}_{h} \mathbf{v}_{A N}+\alpha_{b}^{2} \mathbf{p}_{B F} v_{b}^{\prime},
$$

where $\mathbf{W}_{h} \in \mathbb{C}^{N_{t} \times N_{t}-1}$ stands for an orthonormal basis for the null-space of $\mathbf{h}^{H}$, and for a given ER channel, $\mathbf{p}_{B F}=\mathbf{g} /\|\mathbf{g}\|$. In particular, the artificial noise signal $\mathbf{v}_{A N} \sim$ $\mathcal{C N}\left(0, \mathbf{I}_{N_{t}-1}\right)$ and the energy signal $v_{b}^{\prime} \sim \mathcal{C N}(0,1)$ are transmitted in the null space of the IR's channel and beamformed towards the ER, respectively. While the former provides gains in terms of secrecy, the latter is beneficial from an energy harvesting perspective. The scalars $\alpha_{A N}$ and $\alpha_{b}$ determine the portion of power allocated to each of these signals. The optimization problem can then be stated as

$$
\begin{array}{ll} 
& \max R_{s, l} \\
\text { s.t. } \quad & \operatorname{tr}\left(\mathbf{P}_{D} \mathbf{P}_{D}^{H}\right)+\alpha_{A N}^{2}+\alpha_{b}^{2} \leq N_{t} P_{t x}, \\
& \text { and } \quad E_{H} \geq E_{0},
\end{array}
$$

where the instantaneous harvested energy (conditioned on $\mathbf{g}$ ) is given by

$$
E_{H}=\gamma_{z}\left(\mathbf{g}^{H} \mathbf{P}_{D} \mathbf{P}_{D}^{H} \mathbf{g}+\alpha_{A N}^{2} \mathbf{g}^{H} \mathbf{W}_{b} \mathbf{W}_{b}^{H} \mathbf{g}+\alpha_{b}^{2}\|\mathbf{g}\|^{2}\right) .
$$

In order to solve (31)-(33), we propose a two-stage transmit signal design algorithm. In the first stage, without considering (33), the precoder matrix $\mathbf{P}_{D}$ is obtained using the precoder 
design algorithms given in [9] and [13] for perfect and statistical ECSIT scenarios, respectively. Due to the assumption that the ER works at high SNRs, these algorithms lead to precoders with small $\operatorname{tr}\left(\mathbf{P}_{D} \mathbf{P}_{D}^{H}\right)$ values. Therefore, the remaining power, $P_{\text {rem }}=N_{t} P_{t x}-\operatorname{tr}\left(\mathbf{P}_{D} \mathbf{P}_{D}^{H}\right)$ is divided between the signals $\mathbf{v}_{A N}$ and $v_{b}^{\prime}$ such that the constraints (32) and (33) are satisfied. In order to obtain the portion of power allocated to each artificial noise term, under the perfect or the statistical ECSIT, we perform a one-dimensional parameter search to solve

$$
\begin{gathered}
\min _{\alpha_{b}} \alpha_{b}^{2} \\
\text { s.t. } \\
E_{H}\left(\alpha_{b}\right) \geq E_{0} .
\end{gathered}
$$

By solving (35)-(36), we obtain the minimum power that should be allocated to the artificial noise, which is beamformed to the ER such that the constraint on the harvested power is satisfied. Since this artificial noise leaks into the IR's channel, it is reasonable to find the minimum power that can be allocated to this signal to satisfy (36). The remaining power is allocated for injection of artificial noise in the null-space of the main channel (i.e., $\alpha_{A N}^{2}=P_{r e m}-\alpha_{b}^{2}$ ), and accordingly, the power constraint in (32) is satisfied.

The proposed two-stage precoder design can also be used in the scenarios with statistical ECSIT. In this case, the signal $\mathbf{u}$ is generated using (30) with $\mathbf{p}_{B F}=\mathbf{V}_{\boldsymbol{\Psi}_{1}}$ where $\mathbf{V}_{\boldsymbol{\Psi}_{1}}$ is the first column of the left singular matrix $\mathbf{V}_{\Psi}$, which is the eigenvector corresponding to the largest eigenvalue of $\boldsymbol{\Psi}_{t}$. The relevant optimization problem is posed by replacing $R_{s, l}$ and $E_{H}$ in (31)-(33) by $R_{s, l}^{\prime}$ and $\bar{E}_{H}$, respectively. In particular, in this case, the constraint is on the average harvested power, which is given by

$\bar{E}_{H}=\mathbb{E}_{\mathbf{g}}\left\{\gamma_{z}\left(\mathbf{g}^{H} \mathbf{P}_{D} \mathbf{P}_{D}^{H} \mathbf{g}+\alpha_{A N}^{2} \mathbf{g}^{H} \mathbf{W}_{b} \mathbf{W}_{b}^{H} \mathbf{g}+\alpha_{b}^{2} \mathbf{g}^{H} \mathbf{p}_{B F} \mathbf{p}_{B F}^{H} \mathbf{g}\right)\right\}$

The optimization problem is tackled by taking similar steps with the scenarios with full CSI.

\section{NUMERICAL EXAMPLES}

We now provide numerical examples to examine the efficacy of the proposed algorithms. Throughout the simulations, we model the distance-dependent path loss as

$$
\gamma_{y}=\Gamma_{0}\left(\frac{l_{y}}{l_{0}}\right)^{-\tau} \quad \gamma_{z}=\Gamma_{0}\left(\frac{l_{z}}{l_{0}}\right)^{-\tau},
$$

where $\tau=2.7$ is the path loss exponent and $\Gamma_{0}=10^{-3}$ is the path loss at the reference distance $l_{0}=1 \mathrm{~m}$. We further assume that the ER and the IR's distances from the transmitter are $l_{z}=10 \mathrm{~m}$ and $l_{y}=15 \mathrm{~m}$, respectively. The total transmitted power is $N_{t} P_{t x}=36 \mathrm{dBm}$ and the power of the noise terms at both receivers is $\sigma_{n_{y}}^{2}=\sigma_{n_{z}}^{2}=-50 \mathrm{dBm}$.

First, we focus on a $2 \times 1 \times 1$ set-up with fixed channel coefficients as

$$
\begin{aligned}
& \mathbf{h}^{H}=\left[\begin{array}{ll}
0.5128-0.3239 j & -0.8903-0.0318 j
\end{array}\right], \\
& \mathbf{g}^{H}=\left[\begin{array}{ll}
0.3880+1.2024 j & -0.9825+0.5914 j
\end{array}\right] .
\end{aligned}
$$

Fig. 1 demonstrates the achievable secrecy rates versus the harvested power for different transmission strategies under

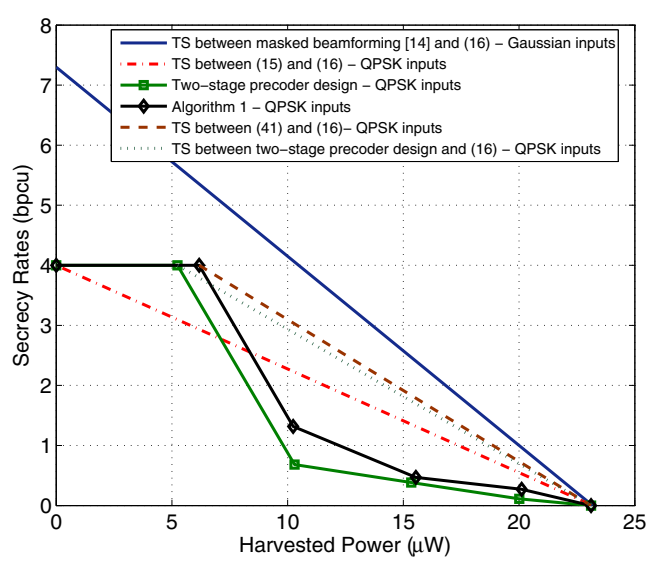

Fig. 1: Achievable secrecy rates versus harvested power with QPSK and Gaussian inputs for the channels given in (39) and (40) and with perfect CSI of both channels at the transmitter.

the perfect CSI assumption. We observe that the proposed transmit signal design algorithm achieves high secrecy rates in the scenarios where the required harvested power is not very high. For example, the solution to the problem in (10)(11) for $E_{0}=6 \mu \mathrm{W}$ is obtained via Algorithm 1 as

$$
\begin{gathered}
\mathbf{P}_{D}=\left[\begin{array}{cc}
0.6403+1.7981 j & -2.0944-2.4498 j \\
-2.5329-0.3699 j & 4.2786-0.9595 j
\end{array}\right], \\
\mathbf{P}_{E}=\left[\begin{array}{cc}
5.2938-54.5471 j & 0 \\
25.3433-17.1919 j & 0
\end{array}\right],
\end{gathered}
$$

which achieves the point $(6.18 \mu \mathrm{W}, 4 \mathrm{bpcu})^{1}$, and time sharing (TS) between this channel input and the one given in (16) considerably outperforms the TS between the strategies introduced in Propositions 1 and 2. Furthermore, it is observed that the low-complexity two-stage design approach yields a comparable performance to that of Algorithm 1 in spite of its significantly reduced complexity. Moreover, the performance gap between the optimal Gaussian and the practical constellation-constrained inputs is clearly observed in Fig. 1.

Fig. 2 demonstrates the trade-off between the ergodic secrecy rates and the average harvested energy for the scenarios where perfect CSI of the IR's channel and only the statistical CSI of the ER are available at the transmitter. We consider a $4 \times 1 \times 1$ setup with BPSK inputs. In order to model the ER's channel, we use (2) and we consider correlation matrices with exponentially decaying entries as

$$
\left[\boldsymbol{\Psi}_{t}(\rho)\right]_{i j}=\rho_{t}^{|i-j|}, \quad i, j=1,2, \ldots, N_{t},
$$

with $\rho_{t}=0.8$. The ergodic secrecy rate and the average harvested energy are obtained via averaging over 500 realizations of the channels. The results demonstrate that both Algorithm 1 and the two-stage precoder design approach offer substantial gains with respect to the TS in this scenario as well.

\footnotetext{
${ }^{1}$ bpcu stands for bits per channel use.
} 


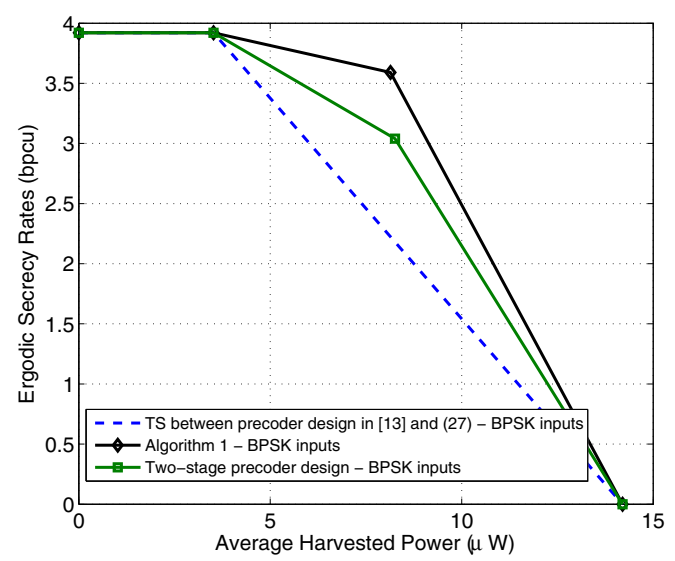

Fig. 2: Achievable ergodic secrecy rates versus average harvested power with BPSK inputs and statistical ECSIT.

\section{CONCLUSIONS}

In this paper, we introduced a framework for transmit signal design and characterized the secrecy rate harvested energy trade-off for the scenarios with multi-antenna transmitters and discrete signaling. The proposed schemes rely on the optimization of the precoder and artificial noise to maximize the achievable secrecy rate given that the harvested energy at the energy receiver is larger than a pre-specified value. Exemplary numerical results demonstrate the efficacy of the proposed solutions.

\section{APPENDIX A \\ DeRIVATION OF $\nabla_{\mathbf{P}_{D}} R_{s, l}$ AND $\nabla_{\mathbf{P}_{E}} R_{s, l}$}

In order to derive $\nabla_{\mathbf{P}_{D}} R_{s, l}$, we use the results in [15] where it has been shown that the gradient of the mutual information expression (e.g., Eq. (5)) can be calculated via the minimum mean-square error (MMSE) matrices $\boldsymbol{\Delta}_{y}\left(\mathbf{P}_{D}\right)$ and $\boldsymbol{\Delta}_{z}\left(\mathbf{P}_{D}\right)$ as

$$
\begin{aligned}
& \nabla_{\mathbf{P}_{D}} I(\mathbf{s} ; y \mid \mathbf{h})=\frac{\log _{2} e}{\sigma_{n_{y}}^{2}+\gamma_{y} \mathbf{h}^{H} \mathbf{P}_{E} \mathbf{P}_{E}^{H} \mathbf{h}}\left(\gamma_{y} \mathbf{h} \mathbf{h}^{H} \mathbf{P}_{D} \boldsymbol{\Delta}_{y}\left(\mathbf{P}_{D}\right)\right), \\
& \nabla_{\mathbf{P}_{D}} I(\mathbf{s} ; z \mid \mathbf{g})=\frac{\log _{2} e}{\sigma_{n_{z}}^{2}+\gamma_{z} \mathbf{g}^{H} \mathbf{P}_{E} \mathbf{P}_{E}^{H} \mathbf{g}}\left(\gamma_{z} \mathbf{g g}^{H} \mathbf{P}_{D} \boldsymbol{\Delta}_{z}\left(\mathbf{P}_{D}\right)\right),
\end{aligned}
$$

where

$$
\begin{aligned}
& \boldsymbol{\Delta}_{y}\left(\mathbf{P}_{D}\right)=\mathbb{E}\left\{(\mathbf{s}-\mathbb{E}\{\mathbf{s} \mid y\})(\mathbf{s}-\mathbb{E}\{\mathbf{s} \mid y\})^{H}\right\} \\
& \boldsymbol{\Delta}_{z}\left(\mathbf{P}_{D}\right)=\mathbb{E}\left\{(\mathbf{s}-\mathbb{E}\{\mathbf{s} \mid z\})(\mathbf{s}-\mathbb{E}\{\mathbf{s} \mid z\})^{H}\right\}
\end{aligned}
$$

The gradient $\nabla_{\mathbf{P}_{D}} R_{s, l}$ is obtained via subtracting (44) from (43).
In order to obtain $\nabla_{\mathbf{P}_{E}} R_{s, l}$, we first use the chain rule as

$$
\begin{gathered}
\nabla_{\mathbf{P}_{E}} R_{s, l}\left(\mathbf{P}_{E}\right)=\sum_{i=1}^{M^{N_{t}}} \mathbb{E}_{n_{y}^{\prime}}\left\{\frac{\sum_{j=1}^{M^{N_{t}}} \exp \left(-\Upsilon_{y, i j}\right)\left(\nabla_{\mathbf{P}_{E}} \Upsilon_{y, i j}\right)}{M^{N_{t}} \ln 2 \sum_{j^{\prime}=1}^{M^{N_{t}}} \exp \left(-\Upsilon_{y, i j^{\prime}}\right)}\right\} \\
-\sum_{m=1}^{M^{N_{t}}} \mathbb{E}_{n_{z}^{\prime}}\left\{\frac{\sum_{k=1}^{M^{N_{t}}} \exp \left(-\Upsilon_{z, m k}\right)\left(\nabla_{\mathbf{P}_{E}} \Upsilon_{z, m k}\right)}{M^{N_{t}} \ln 2 \sum_{k^{\prime}=1}^{M^{N_{t}}} \exp \left(-\Upsilon_{z, m k^{\prime}}\right)}\right\}, \quad \text { (47) }
\end{gathered}
$$

where

$$
\begin{gathered}
\Upsilon_{y, i j}=\frac{\left|\sqrt{\gamma_{y}} \mathbf{h}^{H} \mathbf{P}_{D} \mathbf{d}_{i j}+n_{y}^{\prime}\right|^{2}-\left|n_{y}^{\prime}\right|^{2}}{\sigma_{n_{y}}^{2}+\gamma_{y} \mathbf{h}^{H} \mathbf{P}_{E} \mathbf{P}_{E}^{H} \mathbf{h}}, \\
\Upsilon_{z, m k}=\frac{\left|\sqrt{\gamma_{z}} \mathbf{g}^{H} \mathbf{P}_{D} \mathbf{d}_{m k}+n_{z}^{\prime}\right|^{2}-\left|n_{z}^{\prime}\right|^{2}}{\sigma_{n_{z}}^{2}+\gamma_{z} \mathbf{g}^{H} \mathbf{P}_{E} \mathbf{P}_{E}^{H} \mathbf{g}} .
\end{gathered}
$$

Finally, computing $\nabla_{\mathbf{P}_{E}} \Upsilon_{y, i j}$ and $\nabla_{\mathbf{P}_{E}} \Upsilon_{z, m k}$ using the definition of the complex gradient vector, i.e., $\left[\nabla_{\mathbf{P}_{E}} f\right]_{a b}=\frac{\partial f}{\partial\left[\mathbf{P}_{E}^{*}\right]_{a b}}$, concludes the derivation.

\section{REFERENCES}

[1] X. Chen, D. W. K. Ng, and H.-H. Chen, "Secrecy wireless information and power transfer: challenges and opportunities," IEEE Commun. Mag., vol. 23, no. 2, pp. 54-61, Apr. 2016.

[2] J. Zhang, C. Yuen, C. K. Wen, S. Jin, K. K. Wong and H. Zhu, "Large system secrecy rate analysis for SWIPT MIMO wiretap channels," IEEE Trans. Inf. Forensics Security, vol. 11, no. 1, pp. 74-85, Jan. 2016.

[3] K. Banawan and S. Ulukus, "MIMO wiretap channel under receiver side power constraints with applications to wireless information transfer and cognitive radio, IEEE Trans. on Commun., vol. 64, no. 9, pp. 3872-3885, Sep. 2016.

[4] A. A. Lu, X. Gao, Y. R. Zheng and C. Xiao, "Linear precoder design for SWIPT in MIMO broadcasting systems with discrete input signals: manifold optimization approach," IEEE Trans. on Commun., vol. 65, no. 7, pp. 2877-2888, Jul. 2017.

[5] S. Goel and R. Negi, "Guaranteeing secrecy using artificial noise," IEEE Trans. Wireless Commun., vol. 7, no. 6, pp. 2180-2189, Jun. 2008.

[6] P.-H. Lin S.-H. Lai, S.-C. Lin, and H.-J. Su, "On secrecy rate of the generalized artificial-noise assisted secure beamforming for wiretap channels," IEEE J. Sel. Areas Commun., vol. 31, no. 9, pp. 1728-1740, Sep. 2013.

[7] P. Gopala, L. Lai and H. El Gamal, "On the secrecy capacity of fading channels," IEEE Trans. Inf. Theory, vol. 54, pp. 4687-4698, Oct. 2008.

[8] T. M. Cover and J. A. Thomas, Elements of Information Theory, 2nd Edition. New York, USA: Wiley, 2006.

[9] Y. Wu, C. Xiao, Z. Ding, X. Gao, and S. Jin, "Linear precoding for finitealphabet signaling over MIMOME wiretap channels," IEEE Trans. Veh. Technol., vol. 61, no. 6, pp. 2599-2612, Jul. 2012.

[10] R. Zhang and C. K. Ho, "MIMO broadcasting for simultaneous wireless information and power transfer," IEEE Trans. Wireless Commun., vol. 12, no. 5, pp. 1989-2001, May 2013.

[11] F. Glover, "Surrogate constraint duality in mathematical programming," Operations Research, vol. 23, no. 3, pp. 434-451, 1975.

[12] S. Bashar, Z. Ding, and C. Xiao, "On the secrecy rate of multi-antenna wiretap channel under finite-alphabet input," IEEE Commun. Lett., vol. 15, no. 5, pp. 527-529, May 2011.

[13] S. Rezaei Aghdam, T. M. Duman, "Joint precoder and artificial noise design for MIMO wiretap channels with finite-alphabet inputs based on the cut-off rate," IEEE Trans. Wireless Commun., vol. 16, no. 6, pp. 3913-3923, Jun. 2017.

[14] A. Khisti and G. W. Wornell, "Secure transmission with multiple antennas I: The MISOME wiretap channel," IEEE Trans. Inf. Theory, vol. 56, no. 7, pp. 3088-3104, Jul. 2010.

[15] D. P. Palomar and S. Verdu, "Gradient of mutual information in linear vector Gaussian channels," IEEE Trans. Inf. Theory, vol. 52, no. 1, pp. 141-154, Jan. 2006. 